\title{
Bell's Palsy Cases Following Administration of Influenza A (H1N1) 2009 Monovalent Vaccine Reported to the Vaccine Adverse Event Reporting System (VAERS)
}

\author{
Maria Cano ${ }^{1 *}$, Paige Lewis ${ }^{1}$, Alan C. Ou², Devindra Sharma ${ }^{1}$, Claudia Vellozzi ${ }^{1}$ and Karen R Broder ${ }^{1}$
}

${ }^{1}$ Immunization Safety Office, Division of Healthcare Quality Promotion, National Center for Emerging and Zoonotic Infectious Diseases, Centers for Disease Control and Prevention, USA

${ }^{2}$ Office of Biostatistics and Epidemiology, Center for Biologics Evaluation and Research, Food and Drug Administration, Rockville, MD, USA

\begin{abstract}
Background: With the introduction of 2009-H1N1 vaccine in July 2009, Bell's palsy was one of the adverse events monitored through an enhanced Vaccine Adverse Event Reporting System (VAERS) surveillance during 2009-2010 influenza season.

Methods: We retrospectively reviewed reports to VAERS and available medical records of persons vaccinated with 2009-H1N1 and 2009-2010 seasonal influenza vaccines during July 1- November 30, 2009. We assessed the demographic and clinical characteristics of Bell's palsy patients and the reporting rates of Bell's palsy following these vaccinations.
\end{abstract}

Results: We verified 65 cases Bell's palsy after 2009-H1N1 vaccination and 31 cases after seasonal influenza vaccination. Characteristics of Bell's palsy cases after 2009-H1N1 vaccination were comparable to those after seasonal influenza vaccination and those previously described for Bell's palsy. The overall reporting rate was 2.6 times higher after 2009-H1N1 vaccination than seasonal influenza vaccination but substantially lower than background incidence rates.

Conclusion: There was no pattern in the demographic and clinical characteristics to suggest an increased risk of Bell's palsy after 2009-H1N1 vaccination. The higher reporting rate of Bell's palsy to VAERS following receipt of 2009-H1N1 vaccine compared to seasonal influenza vaccine may be due to stimulated reporting.

\section{Introduction}

With the emergence of pandemic (H1N1) 2009 virus, inactivated influenza A (H1N1) 2009 Monovalent Inactivated Vaccine (MIV) and Live, Attenuated Monovalent Vaccine (LAMV) were developed and distributed in October 2009 in the United States. Trivalent Inactivated Influenza Vaccine (TIV) and trivalent Live, Attenuated Influenza Vaccine (LAIV) was also available to prevent 2009-2010 seasonal influenza. The Advisory Committee on Immunization Practices (ACIP) recommended use of the 2009-H1N1 and seasonal influenza vaccines during the 2009-2010 influenza season [1,2]. The 2009-H1N1 vaccines were expected to have a similar safety profile as seasonal influenza vaccines. However, a comprehensive national 2009-H1N1 vaccine safety monitoring system was established to rapidly detect any unexpected adverse events following 2009-H1N1 vaccination [3]. As part of comprehensive 2009-H1N1 safety monitoring, the Centers for Disease Control and Prevention (CDC) and Food and Drug Administration (FDA) enhanced the Vaccine Adverse Event Reporting System (VAERS) in October 2009 [3,4]. Several outcomes reported to VAERS were closely monitored following 2009-H1N1 vaccination, including Bell's palsy because a 2000-2001 study reported an association between one formulation of TIV (administered intranasally) and Bell's palsy in Switzerland [5]; another study noted a signal of possible association between TIV and increased risk of Bell's palsy in the United States [6]. Here we report the results of enhanced monitoring for Bell's palsy cases reported to VAERS following administration of 2009-H1N1 vaccine.

\section{Methods}

\section{Data source}

VAERS, co-administered by the CDC and FDA, is a national spontaneous reporting surveillance system that accepts reports of adverse events following immunization from healthcare and vaccine providers, manufacturers, and vaccine recipients or their caregivers [7]. The VAERS report form collects information on age, gender, vaccine administered, adverse event experience, and health history. Signs and symptoms of adverse events are coded by trained staff and entered into a database using the Medical Dictionary for Regulatory Activities (MedDRA), an internationally-standardized terminology [8]. Reports are coded as "serious", as defined by the Code of Federal Regulations if at least one of the following was reported: death, lifethreatening illness, hospitalization or prolonged hospitalization, or permanent disability [9]; other reports are coded as "non-serious". Following standard procedure, medical records for all serious nonmanufacturer reports are requested, and physicians at the CDC and FDA reviewed the reported events. Enhanced safety monitoring for 2009-H1N1 vaccines included requesting and reviewing medical records for all (both serious and non-serious) reports of possible Bell's

${ }^{*}$ Corresponding author: Maria V. Cano, MD, MPH, Immunization Safety Office, Division of Healthcare Quality Promotion, National Center for Emerging and Zoonotic Infectious Diseases, Centers for Disease Control and Prevention (CDC), Mailstop D-26, 1600 Clifton Road, Atlanta, GA 30333, USA, Tel: 404718-1214; Fax: 404-639-8834; E-mail: mcano@cdc.gov

Received January 20, 2012; Accepted April 24, 2012; Published April 26, 2012

Citation: Cano M, Lewis P, Ou AC, Sharma D, Vellozzi C, et al. (2012) Bell's Palsy Cases Following Administration of Influenza A (H1N1) 2009 Monovalent Vaccine Reported to the Vaccine Adverse Event Reporting System (VAERS). J Vaccines Vaccin 3:134. doi:10.4172/2157-7560.1000134

Copyright: () 2012 Cano M, et al. This is an open-access article distributed under the terms of the Creative Commons Attribution License, which permits unrestricted use, distribution, and reproduction in any medium, provided the original author and source are credited. 
Citation: Cano M, Lewis P, Ou AC, Sharma D, Vellozzi C, et al. (2012) Bell's Palsy Cases Following Administration of Influenza A (H1N1) 2009 Monovalent Vaccine Reported to the Vaccine Adverse Event Reporting System (VAERS). J Vaccines Vaccin 3:134. doi:10.4172/21577560.1000134

palsy to VAERS. This enhancement was also implemented for reports following 2009-2010 seasonal influenza vaccination.

\section{Case identification and verification}

We conducted a retrospective search of potential Bell's palsy cases from U.S. reports in VAERS, received through January 31, 2010 for persons vaccinated with $2009-\mathrm{H} 1 \mathrm{~N} 1$ or $2009-2010$ seasonal influenza vaccines during the period July 1 through November 30, 2009 when enhanced monitoring for all Bell's palsy cases occurred.

To identify possible Bell's palsy reports, we used the following MedDRA terms: facial paresis, facial palsy, facial nerve disorder, oculofacial paralysis, and VIIth nerve paralysis. We reviewed all VAERS reports, including any available medical records and laboratory reports and verified Bell's palsy. A case was considered verified if: (1) there was a diagnosis of Bell's palsy reported by a healthcare provider on the VAERS form or medical records, or there were signs or symptoms (e.g. , facial paralysis) and treatment for Bell's palsy noted on the VAERS form by a healthcare provider; and (2) there was no evidence on the VAERS form or medical records suggesting involvement of cranial nerves other than cranial nerve VII or another diagnosis (e.g., Guillain-Barré Syndrome [GBS], cerebrovascular accident) to explain the facial paralysis was documented.

\section{Descriptive clinical review}

We described the demographic and clinical characteristics of Bell's palsy cases reported after 2009-H1N1 vaccination (with or without concomitant seasonal influenza vaccine) and after 2009-2010 seasonal influenza vaccination given without $2009-\mathrm{H} 1 \mathrm{N1}$ vaccine. These characteristics included: age, sex, vaccine type, onset interval, risk factors associated with Bell's palsy (e.g., pregnancy, diabetes) and facial side of paralysis.

\section{Reporting rates}

We calculated the reporting rates for verified reports of Bell's palsy. For the denominators, we used the estimated doses administered for four age groups (6 months-17 years, 18-49 years, 50-64 years, and $\geq 65$ years) from October 1 through November 30, 2009 for 2009H1N1 vaccines and August 1 through November 30, 2009 for seasonal influenza vaccines based on vaccine coverage rates obtained from the National 2009 H1N1 Flu Survey and population data from the United States Census 2010 [10-12]. Reports received after simultaneous administration of seasonal influenza and $2009-\mathrm{H} 1 \mathrm{~N} 1$ vaccine were included in the numerators for both vaccine types since the denominator estimates did not differentiate simultaneous vaccinations. We used chisquare statistical test to assess reporting rate differences between $\mathrm{H} 1 \mathrm{~N} 1$ and seasonal influenza Bell's palsy cases; a $p$ value $<0.05$ was considered significant (SAS, version 9.2, SAS Institute, Inc. Cary, NC).

\section{Results}

Medical records were available for 88 (95\%) and 48 (87\%) of reports of potential Bell's palsy following 2009-H1N1 and seasonal influenza vaccinations, respectively. The diagnosis of Bell's palsy was verified in $70.7 \%$ of those reports after receipt of $2009-\mathrm{H} 1 \mathrm{~N} 1$ (with or without concomitant seasonal influenza vaccine) and in $56.4 \%$ of those reports after seasonal influenza vaccination without 2009-H1N1 vaccine (Figure 1). GBS was the most common reported diagnosis among the reports that were determined not to be Bell's palsy after 2009-H1N1 and seasonal influenza vaccinations (Table 1).

Most of the vaccines with Bell's palsy after 2009-H1N1 or seasonal influenza vaccinations received the inactivated formulations of these vaccines (Table 2). A higher proportion of the cases after 2009-H1N1 vaccination received live, attenuated formulation of the vaccine than the cases after seasonal influenza vaccination. Age range and median age were similar for both 2009-H1N1 and seasonal influenza vaccine recipients. For 2009-H1N1 vaccine, the highest proportion of Bell's palsy reports was in the 18-49 years age group. For seasonal influenza vaccine, age groups $\leq 17$ years and $18-49$ years had the highest proportion of Bell's palsy.

Onset interval (from the date of vaccination [day 0] to the date of

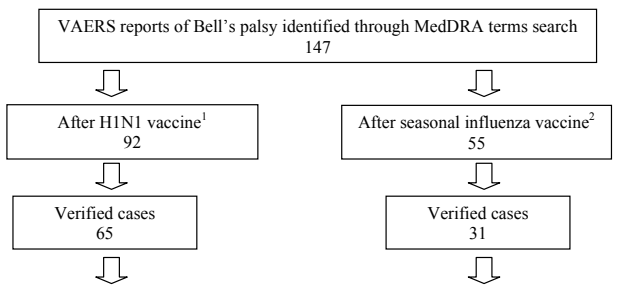
H1N1 vaccine only: 36 ( 28 inactivated, 8 live, attenuated)
H1N1 and seasonal influenza ${ }^{3}: 27$
Seasonal influenza vaccine (imactivated): 24 Seasonal influenza and other vaccine(s) $)^{5}$.

${ }^{1}$ Reports that documented simultaneous seasonal and 2009-H1N1 vaccine administration were included

${ }^{2}$ Reports that documented simultaneous seasonal and 2009-H1N1 vaccine administration were not counted

${ }^{3}$ One report had Human Papillomavirus Vaccine (HPV) vaccine with the seasonal and 2009-H1N1 vaccines

${ }^{4}$ Other vaccines: tetanus-diphtheria-acellular pertussis (Tdap); HPV

${ }^{5}$ Other vaccines: combined diphtheria-tetanus-acellular pertussis, inactivated polio, and Haemophilus influenza type b (DTaP-IPV-Hib) +pneumococcal conjugate vaccine (PCV); varicella+hepatitisB+hepatitis $A+$ measles-mumpsrubella (MMR) +tetanus-diphtheria (Td)

Figure 1: Bell's palsy case identification and verification after vaccination with 2009-H1N1 vaccine and 2009-2010 seasonal influenza vaccine reported to VAERS.

\begin{tabular}{|c|c|c|c|}
\hline \multicolumn{2}{|l|}{ After H1N1 vaccine ${ }^{1}$} & \multicolumn{2}{|c|}{ After seasonal influenza vaccine ${ }^{2}$} \\
\hline Diagnosis & No. & Diagnosis & No. \\
\hline GBS & 7 & GBS & 16 \\
\hline GBS (Miller-Fisher) & 1 & GBS (Miller-Fisher) & 1 \\
\hline GBS, Bell's palsy & 2 & Cerebrovascular accident & 1 \\
\hline Cerebrovascular accident & 3 & Facial neuralgia & 1 \\
\hline Conversion disorder & 2 & Polyneuritis & 1 \\
\hline Transient ischemic attack & 1 & Right facial weakness & 1 \\
\hline $\begin{array}{l}\text { Acute disseminated } \\
\text { encephalomyelitis }\end{array}$ & 1 & Unknown & 3 \\
\hline Cephalgia & 1 & & \\
\hline Neuroblastoma & 1 & & \\
\hline $\begin{array}{l}\text { Left facial sensory loss }\left(2^{\text {nd }} \text { and } 3^{\text {rd }}\right. \\
\text { divisions of trigeminal nerve })\end{array}$ & 1 & & \\
\hline $\begin{array}{l}\text { Angioedema with transient visual } \\
\text { disturbance change }\end{array}$ & 1 & & \\
\hline Dystonia, psychosomatic reaction & 1 & & \\
\hline Neuropathy, anxiety & 1 & & \\
\hline Probable allergic reaction to vaccine & 1 & & \\
\hline Influenza-like illness & 1 & & \\
\hline $\begin{array}{l}\text { Right facial paresthesia of } \\
\text { undetermined cause }\end{array}$ & 1 & & \\
\hline Unknown & 1 & & \\
\hline Total reports & 27 & Total reports & 24 \\
\hline
\end{tabular}

GBS: Guillain Barré Syndrome

${ }^{1}$ With or without concomitant seasonal influenza vaccine ${ }^{2}$ Without 2009-H1N1 vaccine

Table 1: Primary diagnosis of cases that ruled out for Bell's palsy following of 2009-H1N1 and 2009-2010 seasonal influenza vaccination. 
Citation: Cano M, Lewis P, Ou AC, Sharma D, Vellozzi C, et al. (2012) Bell's Palsy Cases Following Administration of Influenza A (H1N1) 2009 Monovalent Vaccine Reported to the Vaccine Adverse Event Reporting System (VAERS). J Vaccines Vaccin 3:134. doi:10.4172/21577560.1000134

Page 3 of 6

reported symptom onset) of Bell's palsy cases ranged from 0-88 days (median, 8 days) after 2009-H1N1 vaccination and 0-62 days (median, 4 days)after seasonal influenza vaccination (Figure 2). Approximately $50 \%$ of cases had an onset interval within 1 week after 2009-H1N1 vaccination, while $\sim 75 \%$ of cases after seasonal influenza vaccination had onset of symptoms within 1 week of vaccination.

Six reports of verified Bell's palsy were coded as serious, three (4.6\%) after MIV, two (3.1\%) after LAMV and one (3.2\%) after TIV. One serious report after MIV reported permanent disability; the other five serious reports documented hospitalization with length of stay from 2-5 days. Patients were hospitalized because other diagnoses were being considered and required further evaluation (e.g., GBS, multiple cranial neuropathies) or they had other symptoms or illnesses (e.g., epistaxis, anemia) unrelated to Bell's palsy that required treatment.

Risk factors that have been associated with Bell's palsy, including pregnancy and diabetes [13-16], were reported in eight reports from recipients of 2009-H1N1 vaccine and two who received seasonal influenza vaccine; all received inactivated vaccines. Of the eight cases after $2009-\mathrm{H} 1 \mathrm{~N} 1$ vaccination, five $(7.7 \%)$ were pregnant at the time of vaccination and three $(4.6 \%)$ had diabetes. For seasonal influenza vaccine, one (3.2\%) of the Bell's palsy cases was pregnant while the other had diabetes. Upper respiratory illness prior to the onset of Bell's palsy was reported in the following cases: two (3.1\%) of each after MIV and LAMV; two (6.2\%) after TIV; and one (3.2\%) after LAIV. Only one case after MIV had a history of Bell's palsy. The subject reported having three previous episodes of Bell's palsy that resolved after 1-2 months and were not temporally associated with prior vaccinations. None of the cases after seasonal influenza vaccination reported prior Bell's palsy.

\begin{tabular}{|l|l|l|}
\hline & $\begin{array}{l}\text { H1N1 Vaccine } \\
\text { N (\%) }\end{array}$ & $\begin{array}{l}\text { Seasonal Influenza } \\
\text { N (\%) }\end{array}$ \\
\hline $\begin{array}{l}\text { Vaccine type } \\
\text { Inactivated } \\
\text { Live, attenuated }\end{array}$ & $\begin{array}{l}49(75.4 \%) \\
16(24.6 \%)\end{array}$ & $\begin{array}{l}26(83.9 \%) \\
5(16.1 \%)\end{array}$ \\
\hline $\begin{array}{l}\text { Sex } \\
\text { Male }\end{array}$ & $20(30.8 \%)$ & $14(45.2 \%)$ \\
Female & $45(69.2 \%)$ & $17(54.8 \%)$ \\
\hline $\begin{array}{l}\text { Age (yrs) } \\
\leq 17\end{array}$ & $2-64[$ median 32] & $0.67-65[$ median 33] \\
\hline $18-49$ & $17(26.2 \%)$ & $11(35.5 \%)$ \\
$50-64$ & $36(55.3 \%)$ & $11(35.5 \%)$ \\
$\geq 65$ & $12(18.5 \%)$ & $50-64(25.8 \%)$ \\
$1(3.2 \%)$
\end{tabular}

${ }^{1}$ With and without concomitant seasonal vaccine; total $\mathrm{N}=65$ ${ }^{2}$ Without $2009-\mathrm{H} 1 \mathrm{~N} 1$ vaccine; total $\mathrm{N}=31$

Table 2: Demographic information and vaccine type of Bell's palsy cases after 2009-H1N1 and 2009-2010 seasonal influenza vaccines.

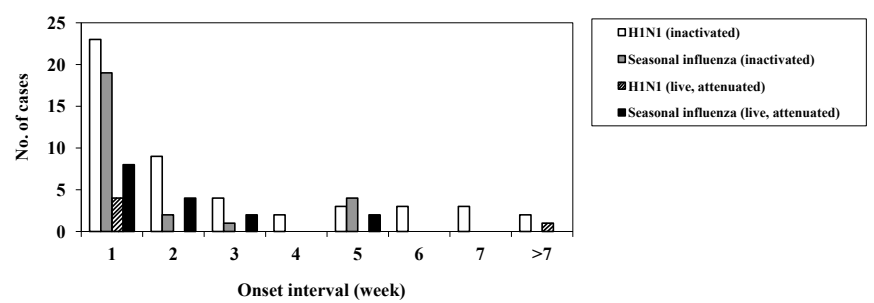

*Reports to VAERS received from July 1, 2009 through January 31, 2010 and vaccinated by November 30, 2009

Figure 2: Onset interval of verified Bell's palsy cases after 2009-H1N1 and seasonal influenza vaccines by vaccine type in the Vaccine Adverse Event Reporting System (VAERS)*.
Among subjects with available information, unilateral facial paralysis was noted in $63(96.9 \%)$ of the Bell's palsy cases after 2009H1N1 vaccination and 27 (96.4\%) after seasonal influenza vaccination. Two $(3.1 \%)$ of the cases after $2009-\mathrm{H} 1 \mathrm{~N} 1$ vaccination and one $(3.6 \%)$ after seasonal influenza vaccination had bilateral facial paralysis.

The reporting rate to VAERS for verified Bell's palsy for all ages was 2.6 times higher after 2009-H1N1 vaccination than 2009-2010 seasonal influenza vaccine (Table 3).The reporting rate was highest in the 18-49 year age group for both 2009-H1N1 and seasonal influenza vaccines, 2.67 vs. 0.94 per million doses received, respectively. Reporting rates were significantly higher for $2009-\mathrm{H} 1 \mathrm{~N} 1$ than seasonal influenza vaccine for age groups 18-49 years and 50-64 years $(\mathrm{p}<0.05)$.

\section{Discussion}

As part of a coordinated vaccine safety surveillance effort during the 2009 (H1N1) pandemic, our study evaluated Bell's palsy in detail following 2009-H1N1 and seasonal influenza vaccinations. Unlike prior studies of Bell's palsy after inactivated influenza vaccination [6,17], our review also included reports after live, attenuated influenza vaccination and compared Bell's palsy reporting after 2009-H1N1 vaccination with seasonal influenza vaccination during the same season. Our study found that verified Bell's palsy cases after 2009-H1N1 vaccination had similar demographic and clinical features to those observed after seasonal influenza vaccination and those previously reported in the literature [18-21].

Our result showed the highest proportion of verified Bell's palsy reports occurred in the 18-64 years age group which was consistent with the findings in a previous review of VAERS data [6]. However, it was inconsistent with population-adjusted reporting rates for Bell's palsy showing the highest rate in $\geq 65$ year age group [6]. This reporting pattern of Bell's palsy reports was observed after both 2009-H1N1 and seasonal influenza vaccinations, although it was more obvious after 2009-H1N1 vaccination. There is no clear explanation for this observation; however in the early stages of the 2009-2010 influenza season, adults $\geq 65$ years of age were not included in the initially recommended priority groups to receive $2009-\mathrm{H} 1 \mathrm{~N} 1$ vaccine [1]. The fact that adults $\geq 65$ years were not vaccinated during the initial part of the vaccination program, when presumably publicity about the H1N1 vaccination program was at its highest, may have impacted reporting practices for this adverse event after both vaccinations. Nationally, seasonal influenza vaccination coverage is expected to be highest among $\geq 65$ years age group [22]. However, during our study period, most of the seasonal influenza doses were administered to the 18-64 years age group. Gender distribution of Bell's palsy after 2009-2010 seasonal influenza vaccination was comparable to those reported in previous studies which showed almost equal frequencies in males and females [19,23]. For 2009-H1N1 vaccine, there was a higher proportion of Bell's palsy among females than males, which might be a result of reporting bias or difference in vaccine uptake.

In the Swiss study that showed an association between intranasal inactivated influenza vaccine and Bell's palsy, more than $50 \%$ of the cases were seen 31-60 days after vaccination [5]. Our study had only one case (6.2\%) with onset interval 31-60 days after vaccination with LAMV, and none had an onset of symptoms in the same period after LAIV. There were nine (18.4\%) cases of Bell's palsy after MIV and three (11.5\%) cases after TIV with onset interval 31-60 days. In a study from the United Kingdom that found no evidence of an increased risk of Bell's palsy following TIV, approximately $30 \%$ of Bell's palsy occurred in each of the periods 1-30 days, 31-60 days and 61-91 post vaccination 
Citation: Cano M, Lewis P, Ou AC, Sharma D, Vellozzi C, et al. (2012) Bell's Palsy Cases Following Administration of Influenza A (H1N1) 2009 Monovalent Vaccine Reported to the Vaccine Adverse Event Reporting System (VAERS). J Vaccines Vaccin 3:134. doi:10.4172/21577560.1000134

Page 4 of 6

\begin{tabular}{|c|c|c|c|c|c|c|c|c|c|c|c|c|c|c|c|c|}
\hline \multirow{3}{*}{ Age group } & \multicolumn{8}{|c|}{ H1N1 vaccine ${ }^{2}$} & \multicolumn{8}{|c|}{ Seasonal influenza vaccine ${ }^{2}$} \\
\hline & \multicolumn{2}{|c|}{ Male } & \multicolumn{2}{|c|}{ Female } & \multicolumn{2}{|c|}{ Overall } & \multirow{2}{*}{$\begin{array}{l}\text { Doses } \\
\text { Administered }{ }^{3} \\
\text { (million) }\end{array}$} & \multirow[t]{2}{*}{ Rate $^{4}$} & \multicolumn{2}{|c|}{ Male } & \multicolumn{2}{|c|}{ Female } & \multicolumn{2}{|c|}{ Overall } & \multirow{2}{*}{$\begin{array}{l}\text { Doses administered } \\
\text { (million) }\end{array}$} & \multirow[t]{2}{*}{ Rate $^{4}$} \\
\hline & No. & $\%$ & No. & $\%$ & No. & $\%$ & & & No. & $\%$ & No. & $\%$ & No. & $\%$ & & \\
\hline$\leq 17$ & 7 & 35.0 & 10 & 22.2 & 17 & 26.2 & 19.0 & 0.89 & 9 & 40.9 & 7 & 19.4 & 16 & 27.6 & 26.3 & 0.61 \\
\hline $18-49$ & 8 & 40.0 & 28 & 62.2 & 36 & 55.3 & 13.5 & $2.67^{*}$ & 8 & 36.4 & 22 & 61.1 & 30 & 51.7 & 31.9 & 0.94 \\
\hline $50-64$ & 5 & 25.0 & 7 & 15.6 & 12 & 18.5 & 6.2 & $1.94^{*}$ & 5 & 22.7 & 6 & 16.7 & 11 & 19.0 & 21.3 & 0.52 \\
\hline$\geq 65$ & 0 & 0 & 0 & 0 & 0 & 0 & 4.7 & 0 & 0 & 0 & 1 & 2.8 & 1 & 1.7 & 22.7 & 0.04 \\
\hline Total & 20 & 100.0 & 45 & 100.0 & 65 & 100.0 & 43.4 & $1.50^{*}$ & 22 & 100.0 & 36 & 100.0 & 58 & 100.0 & 102.2 & 0.57 \\
\hline
\end{tabular}

${ }^{1}$ Reports to VAERS received from July 1, 2009 through January 31, 2010 and vaccinated by November 30, 2009

${ }^{2}$ Simultaneous administration of seasonal influenza and 2009-H1N1 vaccines were included

${ }^{3}$ Based on data from the National 2009 H1N1 Flu Survey and 2010 U. S. Census

${ }^{4}$ Per million vaccine doses administered

*Significant difference $(p<0.05)$ in reporting rates when compared to seasonal influenza vaccine

Table 3: Age and gender distribution and reporting rates by age group of verified Bell's palsy cases following $2009-\mathrm{H} 1 \mathrm{~N} 1$ and $2009-2010$ seasonal influenza vaccines ${ }^{1}$.

[17]. Most of the cases in our study occurred 1-30 days after 2009H1N1 and seasonal influenza vaccinations (both 69\%), which is similar to a prior VAERS study that showed $77 \%$ of Bell's palsy after influenza vaccination with onset of symptoms in the same period [6]. The reporting bias that events closer to the time of vaccination are more likely to be reported may explain the earlier onset intervals in our study and the previous VAERS study compared to the other studies $[5,17]$.

Most of the cases were diagnosed and treated in the outpatient setting, which is consistent with a population-based study that reported the diagnosis of Bell's palsy mainly in a primary care setting [23]. Unilateral facial paralysis was the most common presentation, and other associated symptoms (e.g., paresthesia, numbness, facial weakness, pain behind the ear) were also noted. Paralysis affected the left and right sides of the face with equal frequency. These findings are comparable to the clinical characteristics of Bell's palsy reported in the literature $[13,18]$.

Based on the information in the medical records, it was not possible to confirm the recovery status of the cases after the diagnosis of Bell's palsy. However, even if left untreated, $85 \%$ of cases show improvement within the 3 weeks of onset [24]. Complete recovery has been reported in approximately $70-90 \%$ of cases; recovery was considered moderate in $12 \%$ and poor in $4 \%$ of cases $[20,24]$. Therefore, one report of permanent disability from Bell's palsy among 96 verified cases is not unexpected.

Bell's palsy has been linked to a variety of diseases, including multiple sclerosis, sarcoidosis, and infection with Lyme disease, herpes simplex virus and varicella-zoster virus $[25,26]$. It has also been reported to occur more frequently in persons with prediabetes or diabetes $[13,27]$. Most of the cases in our study did not have any documented prior or concurrent illness at the time of vaccination.

Although Bell's palsy has been reported to occur three times higher in pregnant women than in non-pregnant women [15], other studies did not show an increase during pregnancy $[28,29]$. Of the 340 pregnant women who received 2009-H1N1 vaccine reported to VAERS during our study period, five (1.5\%) had Bell's palsy compared to $24(1.6 \%)$ cases among 1,458 non-pregnant women of reproductive age (i.e., 1544 years) following 2009-H1N1 vaccination [30]. However, among 61 pregnant women who received seasonal influenza vaccine in our study, one (1.6\%) had Bell's palsy compared to six $(0.5 \%)$ cases among 1,126 non-pregnant women of reproductive age following seasonal influenza vaccination.

Higher proportions of Bell's palsy after LAMV vs. LAIV and after TIV vs. MIV may reflect the pattern of influenza vaccines administered. During the 2009-2010 influenza season, 21\% of 2009H1N1 vaccines and $8 \%$ of seasonal influenza vaccines administered were live, attenuated vaccines; $78 \%$ of $2009-\mathrm{H} 1 \mathrm{~N} 1$ vaccines and $93 \%$ of seasonal influenza vaccines were inactivated vaccines [4].

Compared to a study that assessed the background rates of diseases during mass immunization with 2009-H1N1 vaccine [31], reporting rates after $2009-\mathrm{H} 1 \mathrm{~N} 1$ vaccination in our study $(0.89-2.67 /$ million vaccinated) were substantially lower than the expected background incidence rates (19.7-73.8/million vaccinated) of Bell's palsy within 60 days of vaccination. However, our reporting rates were higher than the reporting rate of $0.29 /$ million doses of TIV administered to adults in a review of VAERS data from 1990-2005 [32]. As noted in a study of adverse events following 2009-H1N1 vaccination [4], these findings could reflect stimulated reporting to VAERS. In that study, the reporting rates of all serious and non-serious reports after 2009-H1N1 vaccination were respectively 2.1 and 2.5 times higher than after 20092010 seasonal influenza vaccination, which is comparable to our result. With a newly licensed vaccine against a novel influenza A (H1N1) virus, there may have been a tendency for adverse events to be reported more frequently. There were also numerous efforts to increase 2009H1N1 vaccine adverse events reporting to VAERS [4].

There may also have been some increased reporting of Bell's palsy cases to VAERS after 2009-2010 seasonal influenza vaccination since the rate following receipt of TIV (0.49/million doses) was higher than that reported in VAERS from prior years [6,32]. Reporting rates of Bell's palsy after TIV have varied by influenza season with a range of $0.15-0.46 /$ million doses distributed [6]. Given the variability in reporting rates and use of different denominators to determine rates, these findings should be interpreted with caution.

A substantially increased risk for Bell's palsy was observed after introduction of a Swiss inactivated intranasal influenza vaccine during the 1990s, and the vaccine formulation is no longer in use. The mechanism for the increased risk is not certain, but it has been suggested that Escherichia coli heat labile toxin, used as an adjuvant in the vaccine, may have led to reactivation of latent herpes simplex virus in vaccine recipients [33]. The potential role of the intranasal administration route was also raised as a concern because of its anatomic proximity to the facial nerve [34]. A review of VAERS cases in first year after LAIV identified only one Bell's palsy cases among 460 reports [35]. In this study, we were able to closely evaluate Bell's palsy cases after LAMV and LAIV vaccines, administered by the intranasal route, and found no pattern to suggest a safety concern.

Like all passive surveillance systems, VAERS is limited by stimulated or underreporting, and reports may contain incomplete 
Citation: Cano M, Lewis P, Ou AC, Sharma D, Vellozzi C, et al. (2012) Bell's Palsy Cases Following Administration of Influenza A (H1N1) 2009 Monovalent Vaccine Reported to the Vaccine Adverse Event Reporting System (VAERS). J Vaccines Vaccin 3:134. doi:10.4172/21577560.1000134

or inaccurate data. We partially overcame this limitation by obtaining and reviewing medical records for $>90 \%$ of cases. VAERS is not designed to assess if a vaccine caused an adverse event. Nevertheless, VAERS provides a comprehensive and rapid evaluation and detection of potential adverse events following vaccination. Consistent with a previously published review of VAERS reports following 2009-H1N1 vaccination [4], we focused specifically on Bell's palsy and identified no new safety concerns suggesting an increased risk of Bell's palsy after 2009-H1N1 vaccination. Although the CDC's Vaccine Safety Data link Project detected a signal for an association of Bell's palsy for adults aged $\geq 25$ years following 2009-H1N1 during active surveillance of adverse events, subsequent analyses revealed no significant association [36]. Moreover, recently the Institute of Medicine concluded that evidence favors rejection of a causal relationship between seasonal TIV and Bell's palsy [37].

Our report provides a clinical review of VAERS data following vaccination against influenza virus (H1N1) during the 2009-2010 pandemic and identified no safety concerns. In the event that another novel influenza virus emerges in the future, our evaluation of Bell's palsy cases after vaccination provides a basis of comparison of Bell's palsy following a new influenza vaccine.

\section{Acknowledgments}

We thank the following staff who participated on the CDC-FDA VAERS 2009H1N1 Influenza Response Team: Jorge Arana, MD, MPH, Edward Davis, MSc, Frank DeStefano, MD, MPH, Gary Euler, DrPH, Carolyn Furlow, PhD, Alice Guh, MD, MPH, Laura Kearns, MPH, MPA, Zanie Leroy, MD, MPH, David Martin, MD, MPH, Michael M. McNeil, MD, MPH, Elaine Miller, RN, MPH, Pedro Moro, MD, MPH, Michael Nguyen, MD, MPH, James Singleton, MS, Kamesha Smith, MPH, Jerry Tokars, MD, MPH.

\section{References}

1. National Center for Immunization and Respiratory Diseases, CDC, Centers for Disease Control and Prevention (CDC) (2009) Use of influenza A (H1N1) 2009 monovalent vaccine: recommendations of the Advisory Committee on Immunization Practices (ACIP), 2009. MMWR Recomm Rep 58: 1-8.

2. Fiore AE, Shay DK, Broder K, Iskander JK, Uyeki TM, et al. (2009) Prevention and control of seasonal influenza with vaccines: recommendations of the Advisory Committee on Immunization Practices (ACIP), 2009. MMWR Recomm Rep 58: 1-52.

3. Salmon DA, Akhtar A, Mergler MJ, Vannice KS, Izurieta H, et al. (2011) Immunization-safety monitoring systems for the $2009 \mathrm{H} 1 \mathrm{~N} 1$ monovalent influenza vaccination program. Pediatrics 127: S78-S86.

4. Vellozzi C, Broder KR, Haber P, Guh A, Nguyen M, et al. (2010) Adverse events following influenza A (H1N1) 2009 monovalent vaccines reported to the Vaccine Adverse Event Reporting System, United States, October 1, 2009January 31, 2010. Vaccine 28: 7248-7255.

5. Mutsch M, Zhou W, Rhodes P, Bopp M, Chen RT, et al. (2004) Use of the inactivated intranasal influenza vaccine and the risk of Bell's palsy in Switzerland. N Engl J Med 350: 896-903.

6. Zhou W, Pool V, Destefano F, Iskander JK, Haber P, et al. (2004) A potential signal of Bell's palsy after parenteral inactivated influenza vaccine: reports to the Vaccine Adverse Event Reporting System (VAERS)—United States, 1991 2001. Pharmacoepidemiol Drug Saf 13: 505-510.

7. Varricchio F, Iskander J, Destefano F, Ball R, Pless R, et al. (2004) Understanding vaccine safety information from the Vaccine Adverse Event Reporting System. Pediatr Infect Dis J 23: 287-294.

8. Medical Dictionary for Regulatory Activities.

9. Food and Drug Administration. (2005) 21 CFR Part 600.80. Postmarketing reporting of adverse experiences. Federal Register 70 FR 148982.

10. United States Census 2010
11. Centers for Disease Control and Prevention (CDC) (2010) Interim results state-specific influenza A (H1N1) 2009 monovalent vaccination coverageUnited States, October 2009-January 2010. MMWR Morb Mortal Wkly Rep 59: 363-368.

12. Centers for Disease Control and Prevention (CDC) (2010) Interim results: state-specific seasonal influenza vaccination coverage-United States, Augus 2009-January 2010. MMWR Morb Mortal Wkly Rep 59: 477-484.

13. Tiemstra JD, Khatkhate N (2007) Bell's palsy: Diagnosis and management. Am Fam Physician 76: 997-1002

14. Shmorgun D, Chan WS, Ray JC (2002) Association between Bell's palsy in pregnancy and pre-eclampsia. QJM 95: 359-362.

15. Shapiro JL, Yudin MH, Ray JG (1999) Bell's palsy and tinnitus during pregnancy: predictors of pre-eclampsia? Three cases and a detailed review of the literature. Acta Otolaryngol 119: 647-651.

16. Morris AM, Deeks SL, Hill MD, Midroni G, Goldstein WC et al. (2002) Annualized incidence and spectrum of illness from an outbreak investigation of Bell's palsy. Neuroepidemiology 21: 255-261.

17. Stowe J, Andrews N, Wise L, Miller E (2006) Bell's palsy and parenteral inactivated influenza vaccine. Hum Vaccin 2: 110-112.

18. Gilden DH (2004) Clinical practice. Bell's palsy. N Engl J Med 351: 1323-1331.

19. De Diego JI, Prim MP, Madero R, Gavilan J (1999) Seasonal patterns of idiopathic facial paralysis: a 16-year study. Otolaryngol Head Neck Surg 120 269-271.

20. Peitersen E (1982) The natural history of Bell's palsy. Am J Otol 4: 107-111.

21. Rath B, Linder T, Cornblath D, Hudson M, Fernandopulle R, et al. (2007) All that palsies is not Bell's-the need to define Bell's palsy as an adverse event following immunization. Vaccine 26: 1-14.

22. Centers for Disease Control and Prevention (2010) Final estimates for 200910 seasonal influenza and influenza A (H1N1) 2009 Monovalent Vaccination Coverage-United States, August 2009 through May, 2010.

23. Rowlands S, Hooper R, Hughes R, Burney $P$ (2002) The epidemiology and treatment of Bell's palsy in the UK. Eur J Neurol 9: 63-67.

24. Peitersen E (2002) Bell's palsy: the spontaneous course of 2,500 periphera facial nerve palsies of different etiologies. Acta Otolaryngol Suppl 4-30.

25. Chou CH, Liou WP, Hu KI, Loh CH, Chou CC, et al. (2007) Bell's palsy associated with influenza vaccination: two case reports. Vaccine 25: 28392841.

26. Piercy J (2005) Bell's palsy. BMJ 330: 1374.

27. Bosco D, Plastino M, Bosco F, Consoli A, Labate A, et al. (2011) Bell's palsy: a manifestation of prediabetes? Acta Neurol Scand 123: 68-72.

28. Vrabec JT, Isaacson B, Van Hook JW (2007) Bell's palsy and pregnancy Otolaryngol Head Neck Surg 137: 858-861.

29. Moro PL, Broder K, Zheteyeva Y, Walton K, Rohan P, et al. (2011) Adverse events in pregnant women following administration of trivalent inactivated influenza vaccine and live attenuated influenza vaccine in the Vaccine Adverse Event Reporting System, 1990-2009. Am J Obstet Gynecol 204: 146.e1-7.

30. Moro PL, Broder K, Zheteyeva Y, Revzina N, Tepper N, et al. (2011) Adverse events following administration to pregnant women of influenza A (H1N1) 2009 monovalent vaccine reported to the Vaccine Adverse Event Reporting System. Am J Obstet Gynecol 205: 473. e1-9.

31. Black S, Eskola J, Siegrist CA, Halsey N, Macdonald N, et al. (2009) Importance of background rates of disease in assessment of vaccine safety during mass immunization with pandemic H1N1 influenza vaccines. Lancet 374: 2115-2122.

32. Vellozzi C, Burwen DR, Dobardzic A, Ball R, Walton K, et al. (2009) Safety of trivalent inactivated influenza vaccines in adults: Background for pandemic influenza vaccine safety monitoring. Vaccine 27 : 2114-2120.

33. Couch RB (2004) Nasal vaccination, Escherichia coli enterotoxin, and Bell's palsy. New Engl J Med 350: 860-861. 
Citation: Cano M, Lewis P, Ou AC, Sharma D, Vellozzi C, et al. (2012) Bell's Palsy Cases Following Administration of Influenza A (H1N1) 2009 Monovalent Vaccine Reported to the Vaccine Adverse Event Reporting System (VAERS). J Vaccines Vaccin 3:134. doi:10.4172/21577560.1000134

34. Lewis D, Huo Z, Barnett S, Kromann I, Giemza R, et al. (2009) Transient facial nerve paralysis (Bell's palsy) following intranasal develivery of a genetically detoxified mutant of Escherichia coli heat labile toxin. PLoS One 4: e6999.

35. Izurieta HS, Haber P, Wise RP, Iskander J, Pratt D, et al. (2005) Adverse events reported following live, cold-adapted, intranasal influenza vaccine. JAMA 294: 2720-2725
36. Lee GM, Greene SK, Weintraub ES, Baggs J, Kulldorff M, et al. (2011) H1N1 and seasonal influenza vaccine safety in the vaccine safety datalink project. Am J Prev Med 41: 121-128.

37. IOM (Institute of Medicine) (2011) Adverse effects of vaccines: Evidence and Causality. The National Academies Press, Washington DC, USA. 